\title{
Sensor Network Deployment under Distance Uncertainty with Robust Optimization
}

\author{
http://dx.doi.org/10.3991/ijoe.v11i9.5061 \\ Junfeng Qiao ${ }^{1,2}$, Sanyang Liu ${ }^{1}$, Jianke Zhang ${ }^{3}$ and Yujun $\mathrm{Niu}^{2}$ \\ ${ }^{1}$ Xidian University, Xi'an, China \\ 2 Nanyang Institute of Technology, Nanyang, China \\ ${ }^{3}$ Xi' an University of Posts and Telecommunications, Xi'an, China
}

\begin{abstract}
We consider the sensor deployment problem in the context of distance uncertainty. It is characterized by differentiated arrangement of specific detection probability thresholds at different locations. The problem is formulated as an integer linear programming (ILP) model firstly, aiming at optimizing the number of sensors and their locations. Based on the robust discrete optimization methodology, the uncertain model is transformed into an equivalent ILP problem considering distance uncertainty. The proposed approach can control the tradeoff between optimality and robustness by varying the parameters named protection levels. Uniform and non-uniform event detection probabiliy distributions are considered in the experiment. The results show that, as the distance uncertainty increases, the constraint violation can be avoided in the robust model and the robust solution can provide a significant improvement at the expense of a small loss in optimality when compared to the optimal solution of a deterministic scenario.
\end{abstract}

Index Terms-sensor network; robust optimization; coverage; uncertainty; differential deployment

\section{INTRODUCTION}

With the rapid progress of sensor design and communication technique, sensor networks have been quickly evolving in both research and practical domains in the last few decades $[1,2]$. They have been applied in a wide range of applications such as military surveillance, environmental monitoring, as well as natural disaster relief and health care. As one of the fundamental issues in such a network, coverage is important to determine how well an area of interest is monitored and a service is provided [3]. The placement of sensors is a critical issue for coverage, because it can affect cost and detection capability of a sensor network.

Deployment can be random or pre-determined. When the environment is unknown or hostile, sensor nodes are air-dropped in a random manner. Random deployment is very simple, but the number of nodes to be deployed has to be much larger than what is actually required for the full coverage. In pre-determined deployment, the locations of nodes are specified and thus the quality of service can be provided in terms of coverage. It is mainly applied when sensors are expensive or when their operation is significantly affected by their positions.

Distance between sensors plays an important role in deterministic deployment, which directly determines the accuracy of the node position. However, distance is generally affected by uncertain external factors, such as measurement error, actual interference. The actual performance of a deployment strategy can be severely influenced by distance uncertainty. Thus a key challenge in sensor deployment is to determine an uncertainty-aware sensor field that reduces cost and provides high coverage.

In this paper, we use the robust discrete optimization methodology to deal with distance uncertainty for the deployment problem in sensor networks. Considering that the distance between nodes is uncertain, a deterministic robust deployment model is established and solved. With the distance uncertainty increasing, the robust solution for the problem provides a significant performance improvement at the expense of a small loss in optimality when compared to its primitive algorithm.

The remainder of the paper is organized as follows. We first introduce a literature review of related work in Section 2. In Section 3, the deployment problem is formulated as an integer linear programming problem. Robust optimization methodology is presented in Section 4. In Section 5, computational experiments are considered. We conclude the paper in Section 6.

\section{RELATED WORK}

Up to now, there are extensive research works that have discussed deployment strategies. According to the method adopted, approaches for sensor deployment can be classified into three categories: virtual force based [4, 5], grid-based [6, 7] and computational geometry based [8,9]. In many cases, non-uniform detection requirements must be considered according to the importance of the surveillance area. High detection accuracy is required for sensitive regions, and low detection for less important regions. Consequently, differentiated deployment algorithms should be designed based on the required detection probability thresholds at different locations. Some differentiated strategies [11-14] will be listed as follows.

The grid-based deployment algorithm is presented in Reference [10], and the binary detection model is assumed for simplicity. The strategy deals with the deployment of heterogeneous sensors, that is, their sensing ranges and unit price are different. The authors formulate the problem as an integer linear programming model and utilize a mathematical software tool lpsolve to solve it.

In Reference [11], the authors propose two deterministic deployment algorithms, in which a probabilistic event detection model is assumed. The strategies are based on a grid structure. The first 
algorithm, Max-Avg-Cov, aims to maximize the average coverage of the grid points. The second one, Max-MinCov aims to maximize the coverage of the grid points which are the least effectively covered. Both Max-AvgCov and Max-Min-Cov are initially designed for an area with uniform detection probability.

Another differentiated deployment algorithm named Diff-Deploy is proposed in Reference [12]. As in [11], they assume a probabilistic event detection model. By using a Linear Shift (LSI) Invariant system to represent the relationship between the deployment strategy and the miss probability distribution, the authors construct an integer linear optimization problem and solve it iteratively.

In Reference [13], the differentiated deployment problem is formulated as a combinatorial optimization model and then solved based on the Tabu search metaheuristic and the artificial potential field. In the strategy, a required event detection probability is guaranteed at each point. Furthermore, several objectives such as the cost, monitoring quality, connectivity and lifetime are all considered.

In Reference [14], considering that sensors may not always provide reliable information, the authors define an evidence-based coverage model as a generalization of the probabilistic model. Under this model, the deployment problem is formalized as a combinatorial optimization problem, and an algorithm is designed based on dynamic programming. Such issues as the differentiated deployment, connectivity and cost are discussed in the paper.

In Reference [4], the authors present two uncertaintyaware algorithms for sensor deployment, called MINMISS and MAX-MISS. They use a Gaussian probability distribution to model the deviation of the actual location from the intended location. Thus the algorithm produces the mean position of the sensor instead of the actual location of it. In each step, the overall miss probabilities are calculated over all possible grid locations for a newly introduced sensor. Based on these values, a sensor is deployed at the grid with the maximum or minimum miss probability. Nevertheless, it is used for uniform detection requirements.

The optimality of all above algorithms is guaranteed depending on the accuracy of distance between nodes. However, in many applications, distance is usually uncertain due to measurement error or other reasons. Further, few of them [4, 12] have taken this uncertainty into account. If we consider the existence of uncertain factors, the optimality or even the feasibility of these algorithms is very difficult to guarantee. Therefore, the robustness of algorithms should be involved under uncertain environments.

In practical applications, there are two principal approaches to deal with data uncertainty in optimization, namely stochastic and robust optimization. Stochastic optimization [15] has two main drawbacks, one is that the true probability distribution of uncertain data has to be known, and the other is that the stochastic programming model is difficult to solve, also it will suffer from the curse of dimensionality that afflicts it. In Reference [4] and [12], the probability distribution of uncertain location is supposed as the Gaussian distribution. Nevertheless, we cannot infer the result when the uncertain data follow other distributions or even they are ambiguous or rough.

Robust optimization, on the other hand, does not assume that the probability distribution is known, instead it assumes that the uncertain data reside in a so-called uncertainty set [16]. By transforming the corresponding uncertain optimization model into a deterministic problem called its robust counterpart, the optimal solution of the robust counterpart, namely, robust solution is given when uncertain data changes in the uncertainty set. Robust optimization is popular because of its computational tractability for many classes of uncertainty sets and problem types. It has been widely applied to the domains of inventory and logistics, finance, machine learning, energy systems, scheduling, etc [17-19]. Although the first relevant study dates back to 1973 [20], robust optimization has been mainly developed in the last 15 years. Soyster [20] proposes a linear optimization model to construct a solution that is feasible for all input data in a convex set. This approach, however, tends to find solutions that are too conservative to provide much more optimality. Later, Ben-Tal et al. [21-23] carry out further research on the robust optimization theory and have made significant progress in robust convex optimization. However, as the resulting robust counterparts involve nonlinear problems, such methods cannot be applied to discrete optimization. Bertsimas and Sim [24] propose an approach to control the degree of conservatism in the solution of a discrete optimization model by varying a single parameter, and this approach has the potential to be used especially for combinatorial optimization.

For the first time, the robust optimization theory is used for solving the optimization model of sensor networks in Reference [25], in which the robust convex optimization is applied to continuous variables. When the distance between nodes is uncertain, robust counterparts are given and solved for three problems: minimum energy consumption, maximum data extraction, and maximum network lifetime; experimental results show that the robust optimization model has very good performance in practice. To the best of our knowledge, there has not been any sensor deployment research associated with robust optimization. Consequently, combined with the theory and methodology of robust discrete optimization, this paper will use the approach of [24] to address the distance uncertainty for the deployment problem in sensor networks. Belonging to problem-driven studies, this paper will have great theoretical and practical value in solving the uncertainty problem in applications of sensor networks.

\section{Problem Statement}

\section{A. Sensor Detection Model}

The deployment problem is closely related to the sensor detection model, which can be defined in different forms and is subject to different interpretations, depending on sensor types and application scenarios [3]. Let $j$ be a sensor node, and $i$ be a target in the sensor field. In the binary detection model as shown in Eq.(1), an event of interest $i$ is supposed to be detected by a sensor $j$ if and only if their separating distance is less than the sensing range $R_{s}$. 


$$
t_{i}^{j}= \begin{cases}1 & \text { if } d_{i j} \leq R_{s} \\ 0 & \text { otherwise }\end{cases}
$$

Where $d_{i j}$ denotes the Euclidean distance between a sensor node $j$ and a target located at grid point $i$. Although the binary sensor model is simpler, the uncertainty factors in the measurement are not taken into consideration. A more realistic detection model needs to be expressed in a probabilistic term. In a probability detection model, the target is discovered with a certain probability by several sensors in a cooperative manner. The following model given in Eq.(2) assumes the detection probability of event $i$ by a sensor $j$ follows an exponential decaying function of the distance between the target and the sensor.

$$
t_{i}^{j}=\left\{\begin{array}{cc}
e^{-\alpha d_{i j}} & \text { if } d_{i j} \leq R_{s} \\
0 & \text { otherwise }
\end{array}\right.
$$

Where the parameter $\alpha$ is used to model the quality of the sensor and the rate at which its detection probability diminishes with distance. This model is more realistic than the binary detection model. Therefore, we assume it as our model for the following deployment problem formulation.

\section{B. Problem Formulation}

The sensor field is represented as a two-dimensional $m \times n$ grid of points, denoted by $G=\{1,2, \mathrm{~L}, m \times n\}$. The granularity $l$ of the grid, i.e., the distance between grid points can be adjusted based on a trade-off between the computation time and the precision of the coverage measure. Without loss of generality, we only consider the targets at the grid points. Let $S$ denote the collection of sensors, and we suppose that the sensor nodes are deployed at the grid points, that is $S \subseteq G$. For each sensor, omnidirectional antenna is equipped. Sensor nodes can be assumed to be homogeneous or heterogeneous, that is, their sensing radii $R_{s}$ are able to be identical or not. Furthermore, we consider that $R_{s} \geq \sqrt{2} l$.

Therefore, what we want to solve is the following placement problem: given points of interest $G$ to be monitored, how to determine the collection of sensors $S$, with the minimum number of sensors and their locations obtained so that the detection probability of every grid point exceeds the respective predefined threshold. For each grid point $i$ in $G$, we assume its minimum event detection probability threshold is $t h_{i}$. What is more, we allocate a binary variable $I_{i}$ to indicate whether a sensor is placed at grid $i$.

$$
I_{i}=\left\{\begin{array}{cc}
1 & \text { if a sensor is placed at grid } i \\
0 & \text { otherwise }
\end{array} i \in G\right.
$$

Our objective is to minimize the number of sensors, formally, it can be expressed as

$$
\min \sum_{i \in G} I_{i}
$$

We suppose that the event detection decision is made independently by a sensor. For each grid point $i$ in $G$, its detection probability, denoted by $T_{i}$, is measured by all the neighbor sensors around it, that is

$$
T_{i}=1-\prod_{j \in N_{i}}\left(1-t_{i}^{j}\right)
$$

where $N_{i}$ is the neighbor set of grid $i$, i.e., the collection of sensors located in the circle with a center $i$ and radius
$R_{s} . t_{i}^{j}$ is the detection probability shown by Eq.(2). Eq.(5) is equivalent to

$$
T_{i}=1-\prod_{j \in N_{i}}\left(1-t_{i}^{j}\right)^{I_{j}}
$$

A differentiated deployment exists when different grid points must be covered by different numbers of sensors for preferential monitoring. Therefore, the constraint is that the event detection probability at any grid point $i$ must exceed the threshold $t h_{i}$, and it can be formulated as $T_{i} \geq t h_{i}$.

After a logarithm fetch on this constraint, we have

$$
\sum_{j \in N_{i}} \ln \left(1-t_{i}^{j}\right) I_{j} \leq \ln \left(1-t h_{i}\right) \quad \forall i \in G .
$$

Consequently, our deployment problem can be formalized as follows:

$$
\begin{array}{ll}
\min & \sum_{j \in G} I_{j} \\
\text { s.t. } & \sum_{j \in N_{i}} a_{i j} I_{j} \leq \ln \left(1-t h_{i}\right) \quad \forall i \in G \\
& a_{i j}=\ln \left(1-e^{-\alpha d_{i j}}\right) \\
& I_{j} \in\{0,1\}
\end{array}
$$

Problem (8) is an integer linear programming (ILP) problem. We assume that data uncertainty only affects the distance between the grid nodes and the sensors. In the next section, we will solve this problem using robust optimization methodology.

\section{Robust Optimization Model}

\section{A. Robust Optimization Methodology}

Robust optimization is an effective method for dealing with uncertain data. To introduce this methodology, we consider the following optimization problem under uncertainty:

$$
\begin{aligned}
& \min _{\boldsymbol{x}} f(\boldsymbol{x}, \boldsymbol{u}) \\
& \text { s.t. } \quad g(\boldsymbol{x}, \boldsymbol{u}) \leq 0 \quad \forall \boldsymbol{u} \in U
\end{aligned}
$$

where $\boldsymbol{u}$ is an uncertainty parameter that belongs to the uncertainty set $U$. A robust solution is feasible for all $\boldsymbol{u} \in U$ and optimizes the worst case objection function. In other words, the robust solution is obtained by solving the following robust counterpart problem:

$$
\begin{aligned}
& \min _{\boldsymbol{x}} \max _{\boldsymbol{u}} f(\boldsymbol{x}, \boldsymbol{u}) \\
& \text { s.t. } \quad g(\boldsymbol{x}, \boldsymbol{u}) \leq 0 \quad \forall \boldsymbol{u} \in U
\end{aligned}
$$

The task of robust optimization is to find robust feasible solutions that are feasible for any realization of the uncertain data in the uncertainty set and optimize the objective among these solutions. After constructing a reasonable uncertainty set, the tractable robust counterpart for it should be derived and solved via an exact or approximate approach [21].

\section{B. Model of Data Uncertainty}

In our model, the uncertainty parameter is the distance between the grids and the sensors. Although it is hard or even impossible to know the exact distribution of the distance, we can estimate its mean value and its range in typical applications. Since the logarithmic function is 
monotone increasing, each uncertain constraint coefficient $a_{i j}, i \in G, j \in N_{i}$ in Problem (8) is modeled as an independent and bounded random variable $a_{i j}$ that takes value in $\left[\bar{a}_{i j}-\hat{a}_{i j}, \bar{a}_{i j}+\hat{a}_{i j}\right]$, where $\bar{a}_{i j}$ is called the nominal value of $a_{i j}$, and $\hat{a}_{i j}$ is the maximum deviation from the nominal value. In the worst case, when all the uncertain coefficients change, the robust solution is too conservative.

In order to control the conservatism degree of the robust solution, for every grid point $i$ in $G$, we introduce a protection level $\Gamma_{i}[24]$ for the $i$ th constraint in Problem (8). It is used to control the number of coefficients $a_{i j}, j \in N_{i}$ that are subject to parameter uncertainty, and it takes values in the interval $\left[0,\left|J_{i}\right|\right]$, where $J_{i}=\left\{j \mid \hat{a}_{i j}>0, j \in N_{i}\right\}$. The role of the parameter $\Gamma_{i}$ is to control the tradeoff between the optimality and robustness. Generally speaking, it is unlikely that all of the $a_{i j}, j \in J_{i}$ will change. Our goal is to protect against all cases in which up to $\left\lfloor\Gamma_{i}\right\rfloor$ of these coefficients are allowed to change, and one coefficient $a_{i t}$ changes by at most $\left(\Gamma_{i}-\left\lfloor\Gamma_{i}\right\rfloor\right) \hat{a}_{i t}$.

\section{Robust Optimization Model}

Specifically, the proposed robust counterpart of Problem (8) is as follows [24]:

$$
\begin{aligned}
& \min \sum_{j \in G} I_{j} \\
& \text { s.t. } \max _{\left.\left\{S_{i} \cup\left\{t_{i}\right\}\left|S_{i} \subseteq J_{i},\right| S_{i}|\leq| \Gamma_{i}\right], t_{i} \in J_{i} \backslash S_{i}\right\}}\left\{\sum_{j \in S_{i}} \hat{a}_{i j} I_{j}+\left(\Gamma_{i}-\left\lfloor\Gamma_{i}\right]\right) \hat{a}_{i t_{i}} I_{t_{i}}\right\} \\
& \qquad \sum_{j \in N_{i}} \bar{a}_{i j} I_{j} \leq \ln \left(1-t h_{i}\right) \quad \forall i \in G \\
& \bar{a}_{i j}=\ln \left(1-e^{-\alpha d_{i j}}\right) \\
& I_{j} \in\{0,1\}
\end{aligned}
$$

The selection of $\Gamma_{i}$ depends on the decision-maker's preference for the robustness or the optimality of the solution. If the decision-maker pays more attention to the robustness of the solution, a higher value of $\Gamma_{i}$ should be selected. On the contrary, if the decision-maker pays more attention to the optimality of the solution, a lower value of $\Gamma_{i}$ should be selected.

In order to reformulate Problem (11) as a linear programming model, we obtain [24]

$$
\begin{aligned}
& \min \sum_{j \in G} I_{j} \\
& \text { s.t. } \quad \sum_{j \in N_{i}} \bar{a}_{i j} I_{j}+z_{i} \Gamma_{i}+\sum_{j \in J_{i}} p_{i j} \leq \ln \left(1-t h_{i}\right) \quad \forall i \in G \\
& \bar{a}_{i j}=\ln \left(1-e^{-\alpha d_{i j}}\right) \quad \forall i \in G, j \in N_{i} \\
& z_{i}+p_{i j} \geq \hat{a}_{i j} I_{j} \quad \forall i \in G, j \in J_{i} \\
& p_{i j} \geq 0 \quad \forall i \in G, j \in J_{i} \\
& z_{i} \geq 0 \quad \forall i \in G \\
& I_{j} \in\{0,1\} \quad \forall j \in G
\end{aligned}
$$

Problem (12) is a mixed integer linear programming (MILP) problem. In contrast to its primal problem (8), this robust model introduces new variables $z_{i}$ and $p_{i j}$. While the original Problem (8) involves $|G|$ variables and $|G|$ constraints, its robust counterpart Problem (12) has $2|G|+l$ variables, where $l=\sum_{i=1}^{|G|}\left|J_{i}\right|$ is the number of uncertain coefficients, and $|G|+l$ constraints.

\section{COMPUTATIONAL EXPERIMENTS}

The computational experiments are carried out to compare the performance of the robust solution of Problem (12) with the deterministic solution of Problem (8) without distance uncertainty. For these experiments, we solve Problem (8) and Problem (12) using MATLAB 7.1 and Lingo 11.0. We consider two types of requested event detection probability distributions: one is uniform distribution and the other is non-uniform distribution, that is, the event detection probability threshold at each grid point is identical and different respectively. In the following, we will present the obtained results.

\section{A. Experimental Set $U p$}

Network model used in our simulation is the one as described in section $\square$. We assume that the sensor field is a two-dimensional square area with a side equal to 10 units, and the parameter $\alpha$ in equation (2) is set to 0.2 . For the square area, the number of grid points in each row is the same as that in each column. In the experiments, the number of grid points in each row/column increases from 4 to 20 .

In Problem (8) and Problem (12), the constraint coefficient $a_{i j}$ is supposed to be uncertain, and $a_{i j} \in\left[\bar{a}_{i j}-\hat{a}_{i j}, \bar{a}_{i j}+\hat{a}_{i j}\right]$, where we assume its nominal value $\bar{a}_{i j}$ is induced by a distance measurement algorithm. With the measured distance as the norminal distance, the ratio of the distance deviation to this norminal value is denoted as the distance deviation coefficient $\lambda(\lambda \geq 0)$ below. According to the equation constraint in Problem (8), distance deviation will lead to the deviation of $a_{i j}$. Therefore, $\hat{a}_{i j}$ can be computed according to $\lambda$. In our experiments, the sensing range $R_{s}$ is identical for convenience and is set to $R_{s}=\sqrt{2} l$. As a consequence, there are 8 neighbors at each grid point and the protection level $\Gamma_{i}$ can take its maximum value of 8 .

For a minimization problem, we compute the following ratios that compare the robust and deterministic solutions on the nominal data and their respective deviated data [25]:

$$
\begin{aligned}
& R^{a c}=\frac{R_{s o l}(\overline{\boldsymbol{a}})-D_{s o l}(\overline{\boldsymbol{a}})}{D_{s o l}(\overline{\boldsymbol{a}})}, \\
& R^{w c}=\frac{D_{s o l}(\boldsymbol{a})-R_{s o l}(\boldsymbol{a})}{D_{s o l}(\boldsymbol{a})} .
\end{aligned}
$$

Where

$D_{\text {sol }}(\overline{\boldsymbol{a}})$ : optimal value of the deterministic solution. 
$R_{\text {sol }}(\overline{\boldsymbol{a}})$ : objective value of robust solution in the deterministic scenario.

$D_{\text {sol }}(\boldsymbol{a})$ : objective value of deterministic solution in its worst case.

$R_{\text {sol }}(\boldsymbol{a})$ : optimal value of the robust solution.

Problem (8) is solved for the nominal value $\bar{a}_{i j}$ to get the objective value $D_{\text {sol }}(\overline{\boldsymbol{a}})$, and when the constraint coefficients $a_{i j}$ is uncertain, $R_{s o l}(\boldsymbol{a})$ can be obtained by solving Problem (12). We solve Problem (8) in which the deterministic solution is fixed to determine its worst case for $D_{\text {sol }}(\boldsymbol{a})$. The first ratio $R^{a c}$ quantifies the relative loss of optimality of the robust solution on the nominal data, while the second ratio $R^{w c}$ measures the relative increase of the optimal value of the deterministic solution on the deviated data. Therefore the ratio $R^{w c}$ measures the maximum protection that a robust solution can provide, while $R^{a c}$ is the percent increase in cost for this protection.

\section{B. Uniform Distribution}

We test the two deployment strategies when the event detection probability threshold $t h_{i}$ varies from 0.4 to 0.9 in the whole area. When the distance deviation coefficient $\lambda$ increases, that is, the distance uncertainty is enhanced, the robust solution and deterministic solution are compared.

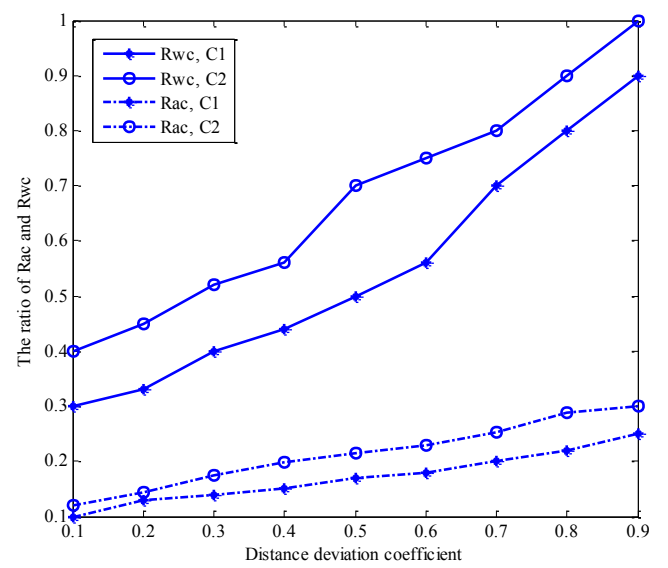

Figure 1. Ratios $R^{a c}$ and $R^{w c}$ for different distance deviation coefficient, scenario $7 \times 7 . t h_{i}=0.9, \mathrm{C}_{1}: \Gamma_{i}=0.2\left|J_{i}\right|, \mathrm{C}_{2}: \Gamma_{i}=0.8\left|J_{i}\right|$.

In Fig. 1 and Fig. 2, the ratios $R^{a c}$ and $R^{w c}$ are presented in different grid dimension of $7 \times 7$ and $14 \times 14$ respectively. In both figures, we illustrate the ratios as a function of the distance deviation coefficient $\lambda$. In Fig. 1, the event detection probability threshold is set to 0.9 and the protection levels $\Gamma_{i}$ of all the grids are set to $0.2\left|J_{i}\right|$ and 0.8 $\left|J_{i}\right|$, denoted as $\mathrm{C}_{1}$ and $\mathrm{C}_{2}$ respectively. As shown in Fig. 1, the ratio $R^{w c}$ is larger than $R^{a c}$ in the two cases. With the increase of $\lambda$, this difference accentuate. Therefore, Problem (12) can provide the solution which exhibits an important improvement under distance uncertainty at the expense of a small loss in optimality. As also can be seen from Fig. 1, a higher value of $\Gamma_{i}$ increases the level of robustness at the expense of lower cost.

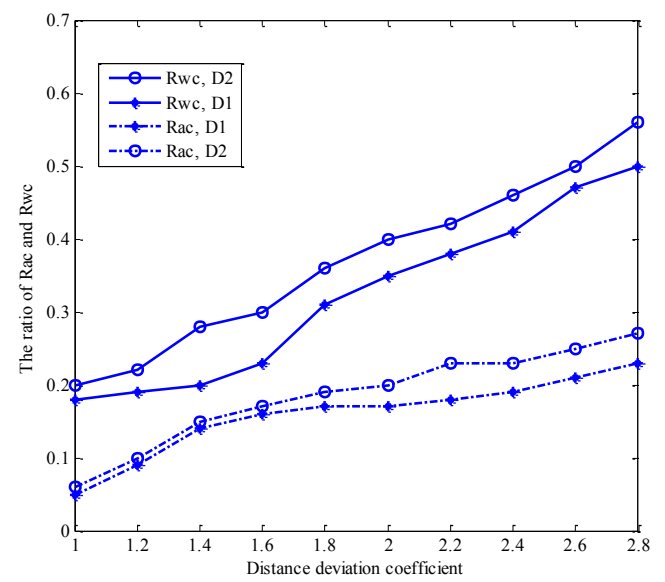

Figure 2. Ratios $R^{a c}$ and $R^{w c}$ for different distance deviation coefficient, scenario $14 \times 14 . \Gamma_{i}=0.8\left|J_{i}\right|, \mathrm{D}_{1}: t h_{i}=0.6, \mathrm{D}_{2}: t h_{i}=0.9$.

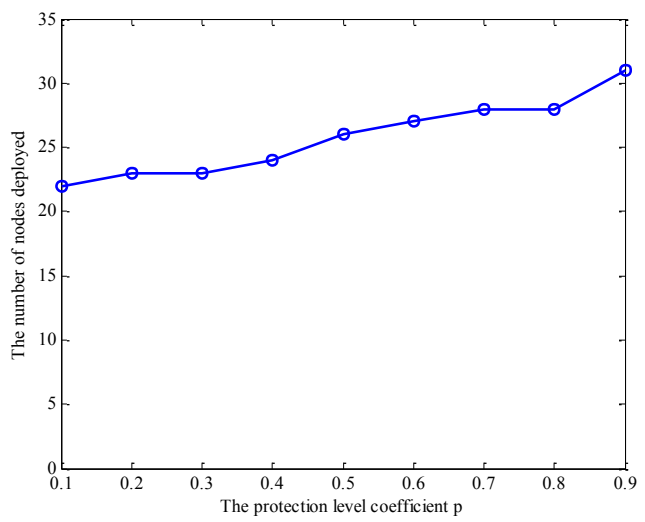

Figure 3. The number of nodes deployed as a function of the protection level $\Gamma_{i}=\rho\left|J_{i}\right|$, scenario $7 \times 7 . t h_{i}=0.9, \lambda=0.5$.

In Fig. 2, we set the protection levels $\Gamma_{i}$ to $0.8\left|J_{i}\right|$ and the event detection probability threshold $t h_{i}$ is set to 0.6 and 0.9 , denoted as $D_{1}$ and $D_{2}$ respectively. Fig. 2 indicates similar results to Fig. 1 in regard to the variation of $R^{w c}$ and $R^{a c}$. The higher the detection probability threshold becomes, the more advantage the robust solution would have.

For the deterministic Problem (8) with $t h_{i}=0.9$, one important thing to note is that the deterministic solution is infeasible for the constraint when $\lambda \geq 1$ in scenario $7 \times 7$, and $\lambda \geq 3$ in scenario $14 \times 14$. However, its robust counterpart can protect it from this constraint violation and provide the robust solution. This observation indicates that robust solution exhibit better performance with the increasing uncertainty. Compared with scenario $7 \times 7$, the distance between grids is shorter in scenario $14 \times 14$. Consequently, distance deviation has a smaller effect on the constraints for more grid points.

As noted by Ben-Tal [21], optimal solutions of linear optimization problems may become severely infeasible if 
the nominal data are slightly perturbed. In realistic scenarios, we would like to accept a suboptimal solution for the nominal values of the data in order to ensure that the solution remains feasible and near optimal when the data changes. The model in Problem (12) is robust with respect to the distance uncertainty and is more practical in real networks.

We next solve the robust Problem (12) by varying the protection levels and report the results in Fig.3, in which $t h_{i}=0.9$ and $\lambda=0.5$. We can see from Fig.3, the number of nodes deployed gradually increases as the protection levels increase, that is, the objective is marginally affected when we increase the protection level.

\section{Non-uniform Distribution}

In this section, we consider an area of $20 \times 20$ grids. We assume that the requested event detection probabilities are non-uniform distributed in the deployment field as shown in Fig. 4. The distribution is illustrated in Fig. 5, and the circled grids denote the locations of deployed sensors. The robust proposal deploys 82 sensors. As seen from Fig. 5, it places sensors according to the requested event detection probability. We also compare the ratios $R^{a c}$ and $R^{w c}$ and the results mirror what we obtain in the previous section. In the interest of space, these results are omitted.

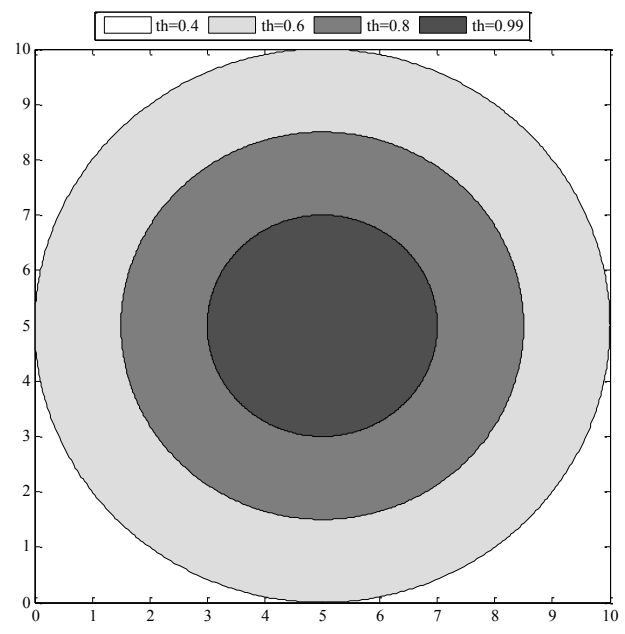

Figure 4. Non-uniform event detection probabilities

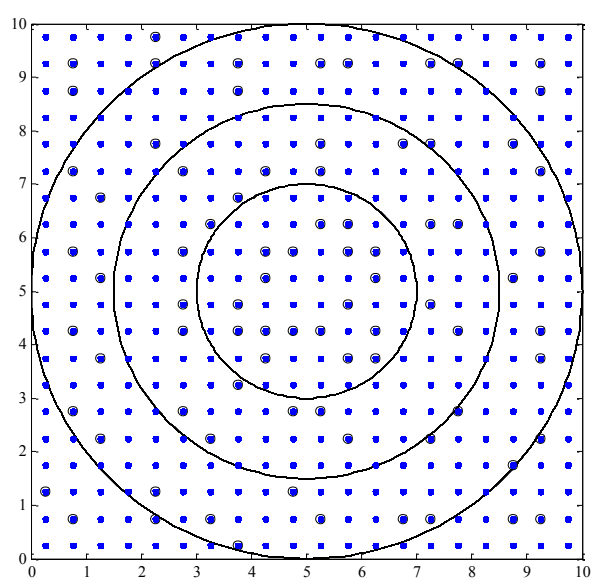

Figure 5. Robust sensor deployment for non-uniform distribution

\section{CONCLUSION}

The efficiency of sensor deployment strategies depends largely on the accuracy of distance between nodes. Unfortunately, distance usually suffers from many uncertain factors, and few deployment problems have taken into account the distance uncertainty. To address this issue, we first formulate the grid-based deployment problem as an integer linear optimization model. Then, by using the 0-1 robust discrete optimization theory, its robust counterpart is obtained and solved. Experimental results show that the tradeoff between the optimality and robustness can be adjusted only by varying the protection levels in the robust model.

This paper has introduced the robust optimization into the deployment technology of sensor networks for the first time. Note that the robust optimization model is not restricted to the detection model we use, other models associated with the distance can also be put to use. The robust discrete optimization theory can also be introduced into other research directions of WSN, such as location technology, routing protocol, and so on. There are still some problems remaining to be solved. Since the ultimate goal of topology control is to provide a better basis for routing, meanwhile to prolong the network lifetime, it is quite necessary to consider routing when dealing with the power control problem. Therefore, the cross-layer power control routing protocols should be designed in the next work.

\section{REFERENCES}

[1] A. Sangwan, R. P. Singh. Survey on coverage problems in wireless sensor networks. Wireless Personal Communications, 2015, 80(4): 1475-1500. http://dx.doi.org/10.1007/s11277-0142094-3

[2] S. Kisseleff, I. F. Akyildiz, W. H. Gerstacker. Throughput of the magnetic induction based wireless underground sensor networks: key optimization techniques. IEEE Transactions on Communications, 2014, 62(12): 4426-4439. http://dx.doi.org/10.1109/TCOMM.2014.2367030

[3] B. Wang. Coverage control in sensor networks. Springer London Ltd, 2010. http://dx.doi.org/10.1007/978-1-84996-059-5

[4] Y. Zou, K. Chakrabarty. Uncertainty-aware and coverage-oriented deployment for sensor networks. Journal of Parallel and Distributed Computing, 2004, 64 (7): 788-798. http://dx.doi.org/10.1016/j.jpdc.2004.03.019

[5] P. M. Pradhan, G. Panda. Connectivity constrained wireless sensor deployment using multiobjective evolutionary algorithms and fuzzy decision making. Ad Hoc Networks, 2012, 10: 1134-1145. http://dx.doi.org/10.1016/j.adhoc.2012.03.001

[6] C. Carmelo, L. Valeria, N. Enrico, R. Tahiry. Nodes selfdeployment for coverage maximization in mobile robot networks using an evolving neural network. Computer Communications, 2012, 35: 1047-1055. http://dx.doi.org/10.1016/j.comcom. 2011.09.004

[7] F. M. Al-Turjman, H.S. Hassanein, M. Ibnkahla. Quantifying connectivity in wireless sensor networks with grid-based deployments. Journal of Network and Computer Applications, 2013, 36(1): 368-377. http://dx.doi.org/10.1016/j.jnca.2012.05.006

[8] C. Lee, D. Shin, S. W. Bae, S. Choi. Best and worst-case coverage problems for arbitrary paths in wireless sensor networks. Ad Hoc Networks, 2013, 11(6): 1699-1714. http://dx.doi.org/10.1016/ j.adhoc.2013.03.005

[9] D. S. Deif, Y. Gadallah. Classification of wireless sensor networks deployment techniques. IEEE Communications Surveys and Tutorials, 2014， 16(2): 834-855. http://dx.doi.org/10.1109/ SURV.2013.091213.00018

[10] K. Chakrabarty, S. S. Iyengar, H. Qi, E.Cho Grid coverage for surveillance and target location in distributed 
sensor networks. IEEE Transactions on Computers, 2002, 51(12): 1448-1453. http://dx.doi.org/10.1109/TC.2002.1146711

[11] S. S. Dhillon, K. Chakrabarty. Sensor placement for effective coverage and surveillance in distributed sensor networks. IEEE Wireless Communications and Networking Conference, New Orleans, LA, United states, 2003: 1609-1614. http://dx.doi.org/10.1109/wcnc.2003.1200627

[12] J. Zhang, T. Yan, S.H. Son, Deployment strategies for differentiated detection in wireless sensor networks. 3rd Annual IEEE Communications Society Conference on Sensor and Ad Hoc Communications and Networks, IEEE, Piscataway, NJ, USA, 2006: 316-325. http://dx.doi.org/10.1109/sahcn.2006.288436

[13] N. Aitsaadi, N. Achir, K. Boussetta, G. Pujolle. Artificial potential field approach in WSN deployment: Cost, QoM, connectivity, and lifetime constraints. Computer Networks, 2011, 55: 84-105. http://dx.doi.org/10.1016/j.comnet.2010.07.017

[14] S. M. Reda, M. Abdelhamid, O. Latifa, A. Amar. Efficient uncertainty-aware deployment algorithms for wireless sensor networks. IEEE Wireless Communications and Networking Conference, IEEE, NY, USA, 2012: 2163-2167. http://dx.doi.org/10.1109/wcnc.2012.6214151

[15] J. R. Birge, F. Louveaux. Introduction to stochastic programming. Springer, New York, 2011. http://dx.doi.org/10.1007/978-1-46140237-4

[16] D. Bertsimas, D. B. Brown, C. Caramanis. Theory and applications of robust optimization. SIAM Review, 2011, 53(3): 464-501. http://dx.doi.org/10.1137/080734510

[17] B. Thomas, B. Christina, D. Fabio, M. Arie, et al. Network planning under demand uncertainty with robust optimization. IEEE Communications Magazine, 2014, 52(2): 178-185. http://dx.doi.org/10.1109/MCOM.2014.6736760

[18] V. Gabrel, C. Murat, A. Thiele. Recent advances in robust optimization: an overview. European Journal of Operational Research, 2014, 235: 471-483. http://dx.doi.org/10.1016/ j.ejor.2013.09.036

[19] B. L. Gorissen, I. Yanikoglu, D. Hertog. A practical guide to robust optimization. Omega, 2015, 53: 124-137. http://dx.doi.org/10.1016/j.omega.2014.12.006

[20] A. L. Soyster. Convex programming with set-inclusive constraints and applications to inexact linear programming. Operations Research, 1973, 21(5): 1154-1157. http://dx.doi.org/10.1287/ opre.21.5.1154

[21] A. Ben-Tal, L. E. Ghaoui, A. Nemirovski. Robust optimization. Princeton, NJ, USA: Princeton University Press, 2009. http://dx.doi.org/10.1515/9781400831050

[22] A. Ben-Tal, D. D. Hertog, D. Waegenaere, B. A. Melenberg, G. Rennen. Robust solutions of optimization problems affected by uncertain probabilities. Management Science, 2013, 59(2): 341357. http://dx.doi.org/10.1287/mnsc. 1120.1641

[23] A. Ben-Tal, D. D. Hertog, J. P. Vial. Deriving robust counterparts of nonlinear uncertain inequalities. Mathematical Programming, 2015, 149(1): 265-299. http://dx.doi.org/10.1007/s10107-0140750-8

[24] D. Bertsimas, M. Sim. Robust discrete optimization and network flows. Mathematical Programming, 2003, 98: 49-71. http://dx.doi.org/10.1007/s10107-003-0396-4

[25] W. Ye, F. Ordonez. Robust optimization models for energylimited wireless sensor networks under distance uncertainty. IEEE Transactions on Wireless Communications, 2008, 7(6): 21612169. http://dx.doi.org/10.1109/TWC.2008.060756

\section{AUTHORS}

Junfeng Qiao is with School of Mathematics and Statistics, Xidian University, Xi'an 710071, China and School of Mathematics and Science, Nanyang Institute of Technology, Nanyang 473004, China. She is currently working toward her $\mathrm{PhD}$ in Xidian University. Now she is an associate professor in Nanyang institute of Technology. Her current research interests include network optimization and wireless sensor networks (e-mail: jfqiao369@126.com).

Sanyang Liu is a professor and $\mathrm{PhD}$ supervisor at the School of Mathematics and Statistics, at Xidian University. His research interest covers theory and application of optimization, network arithmetic (e-mail: liusanyang@126.com).

Jianke Zhang is currently an associate professor at the School of Science, Xi'an University of Posts and Telecommunications, Xi'an 710121, China. His current research interests include robust optimization and intervalvalued programming (e-mail: jiankezh@163.com).

Yujun Niu is an associate professor School of Mathematics and Science, Nanyang Institute of Technology, Nanyang 473004, China. His current research interests include nonlinear system, control and synchronization of complex networks (e-mail: nyjyrff@yeah.net).

This work is supported by the National Natural Science Foundation of China (nos. 61373174, 11302157 and U1504105). Submitted 21 September 2015. Published as resubmitted by the authors 20 October 2015 . 